Sir,

\section{Nocardia keratitis in a corneal graft}

Factors that predispose to the infection of graft following penetrating keratoplasty include suture related problems, persistent epithelial defects, ocular surface disorders, graft failure, use of soft contact lenses, topical steroids and lid abnormalities. Gram-positive cocci and Gram-negative bacilli are associated with majority of the infectious keratitis cases in corneal grafts. ${ }^{1}$ Nocardia as a cause of graft infiltrate is very rare. Colomina $e t \mathrm{al}^{2}$ reported a case of Nocardia peripheral ulcer in a corneal graft patient. We report a case of Nocardia keratitis in a corneal graft being treated for graft rejection with topical steroids.

\section{Case report}

A 25-year-old lady, one year after penetrating keratoplasty for vascularized adherent leucoma in her left eye, developed an episode of endothelial graft rejection. She was being treated with prednisolone acetate $1 \%$ eye drops 12 times a day and ofloxacin $0.3 \%$ eye drops four times a day. The patient was asked to gradually taper the topical steroids over a period of 1 month. However, 2 weeks later, the patient presented with the complaints of increased pain and redness in her left eye of 2 days duration. Best-corrected visual acuity was 20/600. Slit lamp examination revealed conjunctival congestion, graft oedema with descemet's folds and white patchy granular superficial infiltrates in the superonasal quadrant with surrounding diffuse cellular reaction (Figure 1). Anterior chamber showed $1 \mathrm{~mm}$ of hypopyon. With a diagnosis of graft infiltrate, she was subjected to detailed microbiological workup. Gram

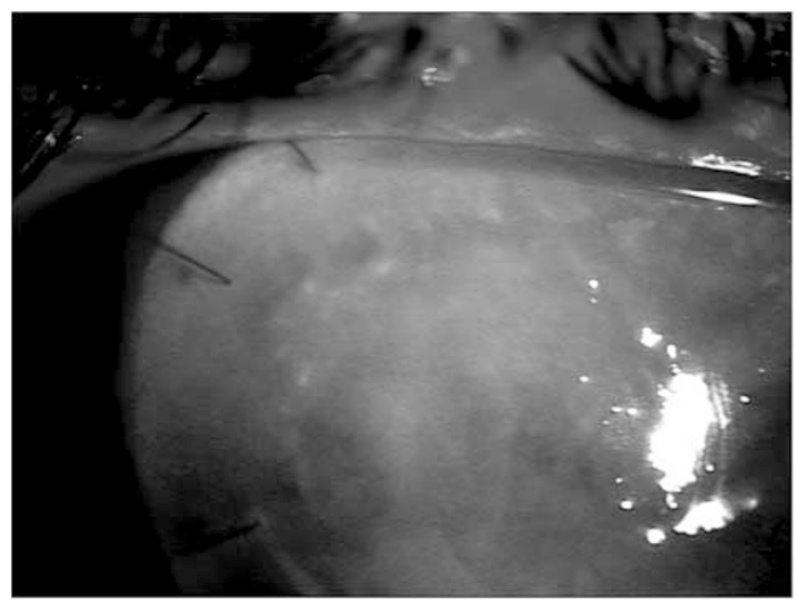

Figure 1 White patchy granular superficial graft infiltrates in the superonasal quadrant with surrounding diffuse cellular reaction.

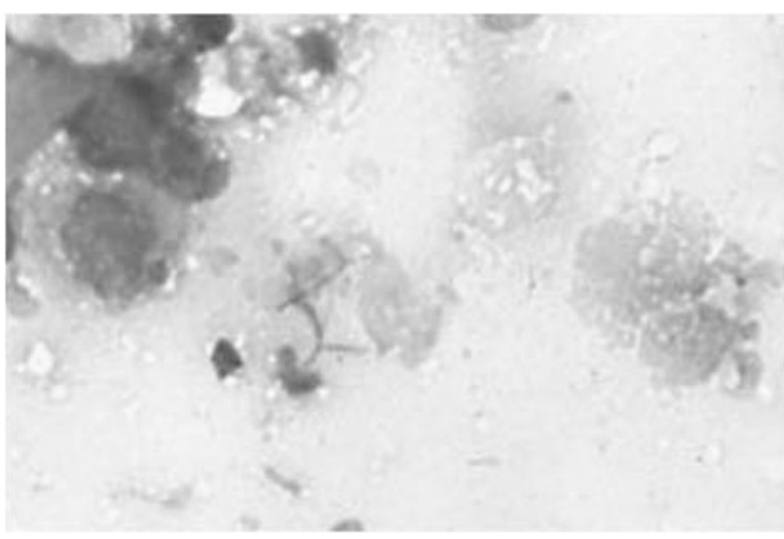

Figure 2 Thin, gram positive, beaded, branching filaments seen in Gram stained smear of the corneal scraping $(\times 500)$.

stained smear revealed thin, Gram positive, beaded branching filaments (Figure 2). The filaments were acid fast when stained with modified Ziehl Neelsen stain using $1 \% \mathrm{H}_{2} \mathrm{SO}_{4}$. There was a significant growth of chalky white colonies on blood agar, subsequently identified as Nocardia asteroides based on its morphology, resistance to decolorization with $1 \%$ sulfuric acid, lack of growth in $0.4 \%$ gelatin and inability to decompose casein, xanthene and hypoxanthene. Antibiotic sensitivity revealed sensitivity to amikacin, ofloxacin and gatifloxacin. Topical steroids were discontinued and the patient was started on topical amikacin $(2.5 \%)$ half hourly, ofloxacin $0.3 \%$ eye drops 2 hourly and atropine sulphate $1 \%$ eye drops 8 hourly. With intensive medical treatment, the corneal infiltrate progressively resolved over a period of 1 month, however, the graft remained oedematous and the vision at last follow up was $20 / 200$.

\section{Discussion}

Nocardia belongs to the order Actinomycetales. It is a Gram positive, weakly acid fast, filamentous saprophyte representing the indigenous microflora of soil and decaying vegetation. Nocardia is not a normal commensal on the ocular surface. Nocardia infections are characterized by an antecedent history of trauma or surgery and run a slow chronic clinical course. ${ }^{3}$ Corticosteroids are known to worsen the infection, probably by stabilizing and inhibiting the release of lysosomal enzymes thereby preventing the destruction of phagocytosed intracellular nocardial organisms. ${ }^{4}$

Our patient was on topical steroids for an episode of graft rejection and probably due to induced local immunosuppression; conditions became favourable for developing Nocardia keratitis. Clinically, the infiltrate was 
not suture related, was superficial and amenable to routine microbiological work up. However, Nocardia may stain irregularly with Gram stain and may fragment into coccobacillary forms leading to a misdiagnosis of Corynebacterium spp. Therefore, acid-fast staining should be a part of the protocol under such circumstances. Owing to varying antibiotic susceptibility, it is essential to determine the drug susceptibility of each isolate. ${ }^{5}$ The infection resolved on treatment with the antibiotic to which the organism was susceptible. However, under such circumstances complete discontinuation of topical steroids, as in our case, may result in graft failure. So, probably a delicate balance is required between continuing low-dose topical steroids and intensive antibiotics to achieve not only the resolution of the infection, but also to prevent graft failure.

Nocardia should be considered as a possible causative agent in a graft infiltrate, especially under local immunosuppression. Resolution of infection can be achieved by early microbiologic workup and initiation of therapy.

\section{References}

1 Tseng SH, Ling KC. Late microbial keratitis after corneal transplantation. Cornea 1995; 14: 591-594.

2 Colomina J, Esparza L, Buesa J, Mari J. Corneal ulcer caused by Nocardia Asteroides after penetrating keratoplasty. Med Clin (Barcelona) 1997; 108: 424-425.

3 Sridhar MS, Gopinathan U, Garg P, Rao GN. Ocular Nocardia infection with special emphasis on the cornea. Surv Ophthalmol 2001; 45: 361-378.

4 Sridhar MS, Cohen E, Rapuano CJ, Lister MA, Laibson PR et al. Nocardia asteroids sclerokeratitis in a contact lens wearer. CLAO J 2002; 28: 66-68.

5 McNeil MM, Brown JM. The medically important aerobic actinomycetes: epidemiology and microbiology. Clin Microb Rev 1994; 7: 358-417.

V Jain' ${ }^{1}$, N Dua ${ }^{1}$, MS Sridhar ${ }^{1}$ and S Sharma ${ }^{2}$

${ }^{1}$ Cornea and Anterior Segment Service, L V Prasad

Eye Institute, L V Prasad Marg, Banjara Hills, Hyderabad, Andhra Pradesh, India

2Jhaveri Microbiology Center, L V Prasad Eye Institute, L V Prasad Marg, Banjara Hills, Hyderabad, Andhra Pradesh, India

Correspondence: S Sharma, L V Prasad Eye Institute, L V Prasad Marg, Banjara Hills, Hyderabad 500 034, Andhra Pradesh, India Tel: +91403061 2502;

Fax: +914023548271.

E-mail: savitri@lvpei.org
Proprietary interest: None

Financial Support: Hyderabad Eye Research

Foundation, Hyderabad, India

Eye (2006) 20, 1385-1386. doi:10.1038/sj.eye.6702221; published online 6 January 2006

Sir,

Closure of macular hole following vitrectomy for diabetic tractional macular detachment

Macular holes in proliferative diabetic retinopathy (PDR) may present an unusual surgical challenge when associated with a macular tractional retinal detachment (TRD). ${ }^{1}$ We successfully closed such a hole, presumed secondary to TRD, using standard pars plana vitrectomy and membrane peeling, without further intervention specifically addressing the macular hole.

\section{Case report}

A 45-year-old insulin-dependent diabetic man was referred with a recent drop in vision of the left eye; best-corrected visual acuity (BCVA) was 6/6 OD and $6 / 36$ OS. Anterior segment examination was unremarkable except for bilateral pseudophakia. Fundus examination revealed postscatter-photocoagulation PDR OU and an extramacular TRD OS. Both eyes underwent additional panretinal photocoagulation. After 2 months, the new vessels regressed; but increasing vitreoretinal traction caused cystoid macular oedema OS. Vitrectomy was advised; but the patient returned only after 4 months, when BCVA had decreased to 2/60 due to macular TRD with a full-thickness macular hole (Figure 1(a)). Optical coherence tomography confirmed the macular hole, and revealed a vitreous membrane bridging the hole (Figure 1(b)). Pars plana vitrectomy, removal of fibrous proliferation, air-fluid exchange, and silicone oil injection were performed without complication. No attempt was made to peel the internal limiting membrane (ILM). After 1 month, left eye had an attached retina and closed macular hole (Figure 1(c)), confirmed by OCT (Figure 1(d)). Silicone oil was removed 6 months later. At 1 year postvitrectomy, significant posterior capsular opacification was noted. After YAG capsulotomy, BCVA improved to $6 / 36$.

\section{Comment}

Macular holes in PDR are caused by fibrovascular traction at the edges of a fovea weakened by oedema and 